\title{
Thermal diffusivity measurement of gold alloys and superconducting ceramics using photoacoustic technique
}

\begin{abstract}
Thermal diffusivity of gold alloys and superconducting ceramics was measured using photoacoustic techniques. All the measurements were carried out at room temperature. Thermal diffusivity of gold alloys was found to be a function of alloy composition. It decreases with the increase in concentration of the doping atoms for superconducting ceramic samples.
\end{abstract}

Keyword: Thermal diffusivity; Gold alloys; Ceramic materials; Photoacoustic techniques 